Vol. 35 (1), 2017 (ISSN 0254-9247)

\title{
Precisión diagnóstica del MMPI-2 con la personalidad delictiva: un análisis con la curva ROC
}

\author{
Amada Ampudia Rueda ${ }^{1}$, Guadalupe Sánchez Crespo ${ }^{2}$, \\ Fernando Jiménez Gómez ${ }^{3}$ \\ Universidad Nacional Autónoma de México ${ }^{1}$, \\ Universidad de Salamanca-España $a^{2,3}$
}

\begin{abstract}
El objetivo de este estudio es poder apreciar la precisión diagnóstica de la personalidad del delincuente mexicano con la prueba del Minnesota Multiphasic Personality Inventory-2 (MMPI-2). Se administró la prueba a un total de 1740 participantes mexicanos de los que 870 (728 varones y 142 mujeres) son reclusos, procesados y/o sentenciados por diferentes delitos, procedentes de diversas cárceles del Estado y Distrito Federal, y otros 870 (728 varones y 142 mujeres) son personas no reclusas. Se utilizó el análisis de la curva ROC (Receiver Operating Characteristic) para apreciar el nivel de precisión diagnóstica a través de sus índices del Área Bajo la Curva (ABC), su Sensibilidad (S) y Especificidad (E). El análisis, diferenciado por género, mostró notables diferencias.
\end{abstract}

Palabras clave: delincuente, MMPI-2, COR, género.

Diagnostic accuracy of the MMPI-2 with the Mexican criminal personality: The ROC curve analysis

The objective of this study is to assess the diagnostic accuracy of the personality of the Mexican criminal with the Minnesota Multiphasic Personality Inventory-2 (MMPI-2). The inventory was administered to 1,740 Mexican participants of which 870 (728 male and 142 female) are prison inmates, processed and/or sentenced for various crimes from various prisons in Mexico City, and the other 870 participants (728 male and 142 female) are not prison inmates. The ROC (Receiver Operating Characteristic) curve analysis was used to assess the level of diagnostic accuracy through indexes of the Area below the curve (ABC),

1 Doctora en Psicología por la Universidad Nacional Autónoma de México. Dirección postal: Av. Universidad, Av. de los Insurgentes Sur, Copilco Universidad, Coyoacán, 04360 Ciudad de México, D.F. Contacto: amada@servidor.unam.mx

2 Doctora en Filosofía y Ciencias de la Educación por la Universidad de Salamanca. Dirección postal: Avda. de la Merced, 101.37005.- Salamanca. Contacto: lupes@usal.es

3 Doctor en Filosofía y Ciencias de la Educación por la Universidad Complutense de Madrid. Dirección postal: Avda. de la Merced, 101.37005.- Salamanca. Contacto: fjimenez@usal.es 
sensitivity (S) and specificity (E). The analysis was differentiated by gender and showed significant differences.

Keyword: Offender, MMPI-2, COR, gender.

Precisão diagnóstica do MMPI-2 na personalidade delitiva mexicana: Uma análise através da curva de ROC

$\mathrm{O}$ objetivo do presente estudo consiste em avaliar a precisão diagnóstica da personalidade do delinquente mexicano através da prova Minnesota Multiphasic Personality Inventory-2 (MMPI-2). A prova foi administrada a 1.740 participantes mexicanos, dos quais 870 (728 homens e 142 mulheres) são reclusos, julgados e condenados por diferentes delitos, procedentes de diferentes estabelecimentos prisionais da cidade do México, e outros 870 (728 homens e 142 mulheres) são pessoas não reclusas. Foi utilizada uma análise da curva de ROC (Receiver Operating Characteristics) para avaliar o nível de precisão diagnóstica através dos índices da Área Abaixo da Curva (ABC), sua sensibilidade (S) e Especificidade (E). A análise de diferenciação entre sexos revelou diferenças significativas.

Palavras chave: delinquente, MMPI-2, COR, género. 
Cada sociedad genera su propia forma de vivir y también de delinquir. La preocupación e interés de los investigadores por la situación de los delincuentes en cárceles mexicanas ha sido una constante desde hace más de una década. Las informaciones aportadas por las diversas encuestas de Azaola (2012), Azaola y Bergman (2009) y Bergman, Azaola y Magaloni (2006), publicadas por el Centro de Investigación y Docencia Económicas (CIDE, 2012), Ampudia (2012), Solís, Buen \& Ley, (2013) y la publicada por la Secretaría de Gobernación (2015) realizadas sobre la situación carcelaria en la que se encuentran los delincuentes mexicanos y su incidencia delictiva, sirven para poner de manifiesto los cuidados y atenciones por parte de la Administración del Centro con los internos encarcelados.

Puede resultar complejo entender el problema de las cárceles de México sin conocer algunos de los problemas más significativos que padece (Gómez-San Luis y Almanza-Avendaño, 2016). En México existen 420 centros penitenciarios y un total de 242,754 internos en dichas instalaciones, con un $95 \%$ son hombres y un $5 \%$ son mujeres, que ocupan un espacio diseñado (cama por recluso) para 195,278, lo cual indica que la ocupación alcanza $124.3 \%$ de sobrepoblación en 220 del total de los 420 centros (Secretaría de Gobernación, enero, 2013). Esta masificación ha sido denunciada reiteradamente por diversos representantes de la Comisión Nacional de Derechos Humanos (Villanueva, 2015) manifestando la necesidad de ser atendida de manera integral por los poderes del Estado estableciendo soluciones y estrategias, políticas, programas y acciones diversas para atender, de manera integral, este problema de hacinamiento (Comisión Nacional de los Derechos Humanos -CNDH- de México, 2015).

Otro problema, no menos latente, a pesar del objetivo propuesto por la legislación existente, es la elevada reincidencia en el delito: "lograr la reinserción social del sentenciado a la sociedad y procurar que no 
vuelva a delinquir" (artículo 18 constitucional). Existe un preocupante incremento en la población de reincidentes que, entre 2005 y 2009, creció un 17\% (Azaola \& Bergman, 2009). Actualmente, de acuerdo con las estadísticas de la Subsecretaría del Sistema Penitenciario (SSP): "de cada 10 internos de los reclusorios de la Ciudad de México que son liberados, cuatro reinciden en la comisión de un delito" (Diario Excelsior, 2016).

Como ocurre en otros muchos países la población penitenciaria mexicana es también básicamente varonil. Únicamente 10,704 internas son mujeres, lo que representa el 4,6\% de la totalidad de la población penitenciaria (Secretaría de Seguridad Pública del Gobierno Federal de los Estados Unidos Mexicanos, octubre de 2011). Esta diferencia, puede estar condicionando y configurando, normativa, política y estructuralmente, unos modelos de cárceles según las necesidades y peculiaridades masculinas (Azaola \& José, 1996) dejando a un lado las necesidades propias femeninas (servicio médico ginecológico, espacios apropiados, cocinas y bańos adecuados para atender y educar a sus hijos). Asimismo, el número de reclusos preventivos y/o sentenciados ha adquirido dimensiones importantes en las cárceles mexicanas: el $56.3 \%$ del total de los internos se encuentran sentenciados, mientras que el $43.7 \%$ son preventivos; es decir, que más de 100,000 personas se encuentran en espera de ser juzgados con la peculiaridad de que algunos podrían resultar absueltos (Llamas, 2013; 2016).

Sabemos de los esfuerzos que la administración y la Secretaría de Seguridad Pública (SSP) mexicana están haciendo por renovar el modelo penitenciario (Estrategia penitenciaria 2008-2012) estableciendo un plan general integrado, no solo de protección a la comunidad sino también del interés por llevar a cabo diferentes tipos de programas de reinserción social para sus internos. Una de las técnicas psicológicas más adecuadas para entender mejor la personalidad del delincuente y el comportamiento de los criminales convictos, tanto para evaluar su estado mental como para evaluar el riesgo de violencia o sus propias necesidades y la posibilidad de beneficiarse de una amplia gama de programas terapéuticos destinados al restablecimiento de sus 
vidas y posibilidad de rehabilitación, es el Cuestionario de Personalidad de Minnesota-2 (MMPI-2). Proporciona varias escalas empíricas que sirven como fundamento y base para la evaluación psicológica de su conducta, en particular las escalas Clínicas básicas de Desviación Psicopática $(P d)$, Paranoia $(P a)$, Esquizofrenia $(S c)$, e Hipomanía $(M a)$ (Butcher, Hess, Greene \& Nelson, 2015).

Es a partir de la década de 1940, cuando el MMPI comenzó a ser considerado como un instrumento eficaz para el examen de los problemas de salud mental y de personalidad en personas que se someten a investigaciones criminales o a ser evaluadas para distintos contextos forenses (Butcher, 2013; Butcher, et al., 2015). Megargee (2006a, 2006b) desarrolló un sistema cuantitativo para la clasificación de los delincuentes adultos mediante perfiles del MMPI. A través del análisis estadístico de clústers seleccionó diez grupos de perfiles para clasificar a los delincuentes (Able, Baker, Charlie, Delta, fácil, Foxtrot, George, Howe, Item, y Júpiter), para poderlos diferenciar según sus característicasde personalidad, aunque su tipología ha sido replicada en numerosos estudios posteriores con diferentes resultados (Butcher, et al. 2015; Megargee, 2006b; Megargee et al. 2009). Tanto el MMPI como el MMPI-2 ha sido empleados para el diagnóstico de una amplia variedad de temas dentro con la población reclusa (Jones, Beidleman \& Fowler, 1981; Lanyon, 1993; Pavelka, 1986; Pennuto, 2010; Steffan, Morgan, Lee \& Sellbom, 2010) para mejor conocimiento y ayudar a las instituciones a mejorar y adecuar su trabajo dentro de las cárceles.

La principal cualidad clínica de una prueba diagnóstica reside en su exactitud, definida como la capacidad para clasificar, de manera correcta, a los individuos en subgrupos clínicamente relevantes. En su forma más simple, es la capacidad para distinguir entre dos estados de salud: sano y enfermo (Burgueño, García-Bastos \& Gonzáles-Buitrago, 1995).

Con el MMPI/MMPI-2, el sistema tradicional para el diagnóstico clínico ha sido la asignación numérica a las 10 escalas clínicas básicas (del 1 al 0) asignando códigos según sea la elevación (punto-uno, puntodos o punto-tres) de sus valoraciones en las distintas variables y cuanto más definido se presentara el código, mayor era su precisión. Con ello 
se pretende identificar los diferentes perfiles de los delincuentes con el objetivo de poder evaluar la salud mental, emitir un informe sobre la asignación de la libertad condicional o establecer las clasificaciones de los internos (Megargee, 2006a, 2006b). El mismo Megargee está preocupado por la fiabilidad en la asignación de los códigos y señala tres inconvenientes sobre este tipo de códigos en los establecimientos penitenciarios: "(a) la falta de fiabilidad en las definiciones operacionales de los tipos de códigos, (b) el hecho de que los perfiles de muchos delincuentes pueden no estar codificados cuando se utilizan criterios estrictos de clasificación, y (c) el hecho de que no haya datos suficientes sobre las correlaciones de tipos de códigos entre los delincuentes" (Megargee, 2006b, p. 339). Esta misma desconfianza de los códigos, hace que sus diferentes tipos de clasificación se basen en un complejo conjunto de reglas en el que considera otros aspectos del perfil y no solo las dos escalas más elevadas en sus puntuaciones (Megargee, 2006b).

Recientemente el análisis de las técnicas de evaluación a través de la curva ROC (Receiver Operating Characteristic) ha sido propuesto como un método discriminante de evaluación para la precisión diagnóstica tras ser una metodología desarrollada en el seno de la Teoría de la Decisión de los años cincuenta (Swets \& Pickett, 1982) e inicialmente diseñada para la detección de señales por radar y, posteriormente, aplicada en el área de la biomedicina (Zweig \& Campbell, 1993). En este estudio se ha utilizado también esta metodología, aportada por la curva ROC, para detectar la precisión diagnóstica del MMPI-2 con los conjuntos de variables psicológicas integrantes en la prueba. Aunque no son frecuentes este tipo de análisis, no obstante, los investigadores cada vez utilizan más esta metodología para poder evaluar la precisión diagnóstica con el MMPI/MMPI-2 (Burgueño, et al. 1995; Jiménez, Sánchez \& Tobón, 2009; Jiménez, Sánchez \& Ampudia, 2009; Nicholson, Mouton, Bagby \& Buis, 1997; Sánchez y Jiménez, 2003; Sánchez, Jiménez y Ampudia, 2008).

La curva ROC, a través de los datos aportados, fundamentalmente, por el Área Bajo la Curva (ABC) la Sensibilidad y la Especificidad, ofrece una visión global de la exactitud diagnóstica proporcionando 
datos significativos acerca de la probabilidad de clasificar correctamente a un individuo mediante una variable determinada. La curva ROC es un gráfico en el que se observan todos los pares Sensibilidad/Especificidad resultantes de la variación continua de los puntos de corte en todo el rango de resultados observados. En el eje de ordenadas se sitúa la Sensibilidad, o fracción de verdaderos positivos, y en el eje X se sitúa la fracción de los falsos positivos (1-Especificidad) (Burgueño, et al. 1995).

El objetivo planteado en este estudio es, mediante el análisis de la curva ROC, poder apreciar la precisión diagnóstica de las diferentes variables contenidas en el MMPI-2 que pueden caracterizar e identificar a la personalidad delictiva.

\section{Método}

El diseño empleado en este estudio es de tipo cuasiexperimental con dos grupos, delincuentes encarcelados y no delincuentes.

\section{Participantes}

La selección de los participantes fue aleatoria dentro de su grupo respectivo de una población más extensa, para poder igualar la muestra por grupos y también por géneros. A los dos grupos se les ha administrado la prueba del Minnesota Multiphasic Personality Inventory-2 (MMPI-2) para poder obtener la precisión diagnóstica mediante el análisis de la curva ROC (Receiver Operating Characteristic).

El número de personas que participaron en este estudio, delincuentes y no delincuentes, fue de 1,740 con un rango establecido entre los 18 y 67 ańos $(M=27.98 ; D E=9.30)$, a quienes se les administró el test de Minnesota Multiphasic Personality Inventory-2 (MMPI-2) para identificar la precisión diagnóstica en su adaptación mexicana (Lucio, Reyes-Lagunes \& Scott, 1994).

Del total de participantes, 870 son delincuentes internados en diferentes cárceles mexicanas del Distrito Federal y del Estado Mexicano de 
los que $728(83.68 \%)$ son varones con un rango de edades entre 19 y 67 ańos $(M=33.72 ; D E=8.44)$, Y $142(16.32 \%)$ son mujeres, con un rango de edad establecido entre 19 y 63 años $(M=32.39$; $D E=7.99)$ Los delincuentes se encuentran internados en diferentes reclusorios: Reclusorio Preventivo Varonil Norte, Reclusorio Preventivo Varonil Oriente, Reclusorio Santa Martha Acatilla, Reclusorio Preventivo Varonil Sur y Centro Varonil de Reinserción Social Santa Martha Acatilla.

El tipo de delito más frecuente por el que están condenados es, en los varones, la posesión/tráfico de drogas (24.45\%), seguido del homicidio (16.07\%), el secuestro (14.29\%), agresión (15.25\%) y robo (13.19\%); sin embargo, las mujeres se encuentran condenadas por robo $(36.62 \%)$, agresión $(33.10 \%)$, homicidio $(27.46 \%)$ y posesión/ tráfico de drogas $(2.82 \%)$.

Con respecto al último nivel de estudios alcanzado, casi la mitad de los varones delincuentes (46.43\%) terminaron la secundaria, el $27.47 \%$ finalizaron el bachillerato, el $16.62 \%$ tienen un trabajo profesional y solamente el $7.55 \%$ finalizaron la primaria. Por el contrario, las mujeres delincuentes el 35.21\% terminaron la secundaria, el $25.06 \%$ realiza un trabajo profesional, el $20.42 \%$ terminaron el bachillerato y un $16.90 \%$ pudieron finalizar la primaria.

Con respecto al estado civil, casi la mitad (42.31\%) de los varones delincuentes están casados, un $27.20 \%$ se encuentran solteros, un $19.23 \%$ se encuentran emparejado en "unión libre", un $5.91 \%$ se encuentran separados, el $4.26 \%$ divorciados y solamente un $1.10 \%$ están viudos. Por el contrario, la mujer reclusa, un 30.28\% se encuentran solteras, un $26.76 \%$ están casadas, un $21.83 \%$ emparejadas en "unión libre", separadas se encuentran un $11.97 \%$, viudas $5.63 \%$ y divorciadas solo el $3.52 \%$.

El grupo de no delincuentes se encuentra referido, también, por un total 870 participantes, de los que 728 (83.68\%) son varones con un rango de edad entre 18 y 59 años $(M=22.48 ; D E=6.68)$; y 142 $(16.32 \%)$ son mujeres, con un rango de edades establecido entre los 19 y 45 ańos $(M=22.44 ; D E=5.41)$. Con respecto al nivel de 
estudios finalizados, más de la mitad (64.42\%) terminaron el bachillerato, un $17.17 \%$ dispone de un trabajo profesional, un $14.15 \%$ terminó la secundaria. Las mujeres no delincuentes el $71.83 \%$ finalizaron el bachillerato, un $12.68 \%$ tenían un trabajo profesional, un 9.86\% habían finalizado el bachillerato y solamente un 5.63\% tenían el nivel de posgrado. Con respecto al estado civil, una amplia mayoría $(85.58 \%)$ de los varones no delincuentes se mantienen solteros, un $13.05 \%$ están casados, en unión libre, $1.24 \%$, y separado, el $0.14 \%$. Las mujeres no delincuentes presentan unas cifras semejantes: $70.42 \%$ se encuentran solteras, el $24.65 \%$ están casadas, unión libre, $1.41 \%$ y divorciadas, un $3.52 \%$.

\section{Instrumentos}

Para poder llevar a cabo el perfil psicológico nos hemos servido del Cuestionario de Personalidad de Minnesota-2 (MMPI-2) en su adaptación mexicana de Lucio et al. (1994) con su conjunto de Escalas Clínicas Básicas, de Contenido y Suplementarias.

\section{Procedimiento}

Con los debidos permisos obtenidos de la Judicatura y de las autoridades regentes en cada centro penitenciario, para realizar esta investigación, se consiguió entrevistar a los internos con el objetivo obtener información (psicológica) que ayude a conocerlos mejor. Algunos protocolos fueron administrados de forma personal o en pequeños grupos, según la disponibilidad de los recursos que se tenían dentro del Centro. Todos los participantes contestaron al cuestionario MMPI-2 de forma voluntaria y libremente aceptada.

Uno de los problemas latentes con los delincuentes es la credibilidad de la información que reportan, ya que el mismo contexto carcelario impide ofrecer una información sincera y veraz por las "consecuencias" que pudieran tener. Al trabajar en este contexto seguimos las recomendaciones seńaladas por Megargee (2006a) acerca de los puntos de corte del MMPI-2 para detectar protocolos incompletos, respuestas aleatorias o incoherentes, fundamentalmente con las escalas de validez 
VRIN y TRIN, antes que con las $F$ (Infrecuencia), $L$ (Mentira) y $K$ (Defensividad). Se descartaron todos aquellos protocolos que el número de ítems No-sé/No-contesta (?) $\geq 30, \mathrm{~L} \geq 80 \mathrm{~T}, \mathrm{~F} \geq 90 \mathrm{~T}, \mathrm{Fb} \geq$ $80 \mathrm{~T}$ y $\mathrm{K} \geq 80 \mathrm{~T}$ y que los valores de VRIN y/o TRIN estuvieran entre 65T-79T (inclusive). Esto supuso la anulación de casi la mitad de los protocolos (40.24\%) de los delincuentes y del $17.38 \%$ de los no delincuentes. Con ello se alcanzó mayor fiabilidad y validez en las respuestas al MMPI-2. Todas las puntuaciones obtenidas fueron baremadas, y tratadas en sus análisis estadísticos, en puntuaciones " $T$ " de acuerdo con la baremación mexicana del MMPI-2. De forma totalmente aleatoria, se igualó el número de participantes, tanto por género como por situación reclusa/no reclusa, de un conjunto superior a los 4000 protocolos MMPI-2. Las muestras fueron obtenidas durante los años 2007-2012.

En el conjunto de variables suplementarias se ha seguido la pauta de prescindir de la escala de detección del trastorno de estrés postraumático de Schlenger (PS) por haber dos escalas del mismo trastorno y por ser anulada en los recientes manuales de Butcher et al. (2015) y Greene, (2011) dejando únicamente la de Keane $(P K)$.

\section{Resultados}

Los datos obtenidos se encuentran expuestos en este estudio, en primer lugar, con el conjunto de género y, posteriormente, se presentan diferenciados por varones y mujeres. En las tablas presentadas en este estudio se muestran los tres estadísticos principales aportados por este estudio: Área Bajo la Curva (ABC), Sensibilidad (S) y Especificidad (E).

\section{Análisis conjunto (ambos sexos)}

La Tabla 1 hace referencia al conjunto de ambos sexos y sus datos se encuentran diferenciados en tres bloques según los tres grupos de variables del MMPI-2: Clínicas, de Contenido y Suplementarias. 
Precisión diagnóstica del MMPI-2 con la personalidad delictiva / Ampudia et al.

\section{Tabla 1}

Precisión diagnóstica de las variables más representativas (MMPI-2) de la personalidad delictiva mexicana. Ambos sexos $(n=870)$

\begin{tabular}{ccccccc}
\hline Variables & ABC & SE & $95 \%$ CI & P. de corte & S. & E. \\
\hline Pd & .760 & .011 & $.739-.780$ & $>51$ & 63.2 & 76.0 \\
Pa & .719 & .012 & $.697-.740$ & $>53$ & 53.4 & 78.9 \\
Si & .742 & .012 & $.720-.762$ & $>44$ & 69.3 & 66.6 \\
D & .700 & .012 & $.678-.721$ & $>49$ & 56.8 & 74.8 \\
Sc & .673 & .013 & $.651-.695$ & $>51$ & 46.9 & 82.3 \\
& .771 & .011 & $.750-.790$ & $>48$ & 61.1 & 80.6 \\
DEP & .773 Clínicas básicas \\
HEA & .709 & .012 & $.687-.730$ & $>48$ & 73.4 & 59.0 \\
FAM & .663 & .013 & $.640-.685$ & $>45$ & 55.7 & 68.5 \\
TRT & .704 & .012 & $.682-.726$ & $>46$ & 64.8 & 66.6 \\
ANX & .680 & .013 & $.658-.702$ & $>51$ & 41.3 & 85.6 \\
SOD & .712 & .012 & $.690-.733$ & $>44$ & 69.2 & 63.1 \\
PK & .729 & .012 & $.707-.749$ & $>44$ & 69.1 & 64.0 \\
Mt & .677 & .013 & $.655-.699$ & $>47$ & 51.6 & 73.9 \\
A & .690 & .013 & $.667-.711$ & $>48$ & 49.4 & 80.6 \\
GM & .821 & .098 & $.802-.839$ & $\leq 53$ & 75.5 & 74.5 \\
\hline
\end{tabular}

Notas: $\mathrm{ABC}=$ Área Bajo la Curva; $\mathrm{SE}=$ Error estándard; $\mathrm{CI}=$ Intervalo de confianza; $\mathrm{P}$. de corte $=$ Punto de corte; S. = Sensibilidad; E. = Especificidad. Pd = Desviación psicopática; $\mathrm{Pa}=$ Paranoia; $\mathrm{Si}=$ Introversión social $; \mathrm{D}=$ Depresión $; \mathrm{Sc}=$ Esquizofrenia $; \mathrm{DEP}=$ Depresión; HEA = Preocupación por la salud; FAM = Problemas familiares; TRT = Indicación negativa hacia el tratamiento; $\mathrm{ANX}=$ Ansiedad; $\mathrm{SOD}=$ Desajuste social; $\mathrm{PK}=$ Trastorno de estrés postraumático (Keane); $\mathrm{Mt}=$ Malestar escolar; $\mathrm{A}=$ Ansiedad, $\mathrm{GM}=$ Rol masculino.

En la Tabla 1, cuando se analizan los datos aportados por el conjunto de delincuentes (varones y mujeres), los valores más elevados mostrados por el Área Bajo la Curva (ABC) son: la variable $G M$ 
(Rol masculino), DEP (Depresión), Pd (Desviación Psicopática), Si (Introversión social), $\mathrm{Pa}$ (Paranoia), $P K$ (Trastorno de Estrés Postraumático), SOD (Desajuste social), HEA (Preocupación por la salud), TRT (Negatividad al tratamiento), y $D$ (Depresión) que son las variables que mejor definen a la personalidad delictiva mediante el MMPI-2. En su interpretación, p. e., un valor de la variable $P d,(A B C=0.76)$, nos está indicando que un individuo, seleccionado aleatoriamente del grupo de delincuentes, tendrá la probabilidad del $76 \%$ de obtener un valor de la variable $P d$ del MMPI-2 mayor que un individuo elegido al azar del grupo de no delincuentes. Para Swets (1979), la capacidad de discriminación de una prueba puede evaluarse estimando el intervalo de confianza (CI) de la $A B C$. Si el intervalo no incluye el valor de 0.5 , la prueba es capaz de discernir entre delincuentes y no delincuentes (en este caso: 0.739-0.780).

El valor de la Sensibilidad y la Especificidad de una prueba va a depender del punto de corte seleccionado. El expresado en las tablas expuestas por el mismo programa informático (MedCalc, 2014) elige el equilibrio entre los valores más elevados de la Sensibilidad y la Especificidad. La Sensibilidad de una prueba diagnóstica es la probabilidad de clasificar correctamente a un individuo como verdadero positivo de una determinada característica (p. e. delincuente). La Especificidad, por el contrario, es la probabilidad de clasificar correctamente a un individuo como verdadero negativo de una determinada característica (p. e. no delincuente).

Con los datos aportados por la Tabla 1, los delincuentes mexicanos de ambos sexos presentan una precisión global de la prueba en torno al $0.70(A B C)$ con un rango entre $0.821(G M)$ y $0.663(F A M)$, considerado por Swets (1979) como una precisión "moderada". En esta Tabla 1 no se muestran las variables MMPI-2 que ha resultado tener un valor de $A B C<0.65$ ya que la ausencia de precisión, por convenio, se encuentra establecido en 0.5 (coincidente con la diagonal de las figuras expuestas).

Las figuras 1 (escalas Clínicas básicas), 2 (escalas de Contenido) y 3 (escalas Suplementarias) muestran, de forma gráfica, la generación 
de la curva ROC según los puntos de corte que se hayan considerado, variando con ello la Sensibilidad y la Especificidad de cada variable.

\section{Análisis por género}

En la Tabla 2 se compara la precisión diagnóstica diferenciada por género. Se ha tomado como referencia que el valor de la $A B C \geq 0.70$ en las mujeres y poder comparar con el valor obtenido por los varones. Se observan dos aspectos interesantes de la precisión diagnóstica del MMPI-2: (1) ha aumentado el número de variables del MMPI-2 que han superado el límite propuesto por la $A B C(\geq 0.70)$ con respecto a las obtenidas cuando el género se evaluaba conjuntamente (Tabla 1); y (2) la existencia de una mayor precisión diagnóstica en las mujeres ( $A B C$, Sensibilidad y Especificidad).

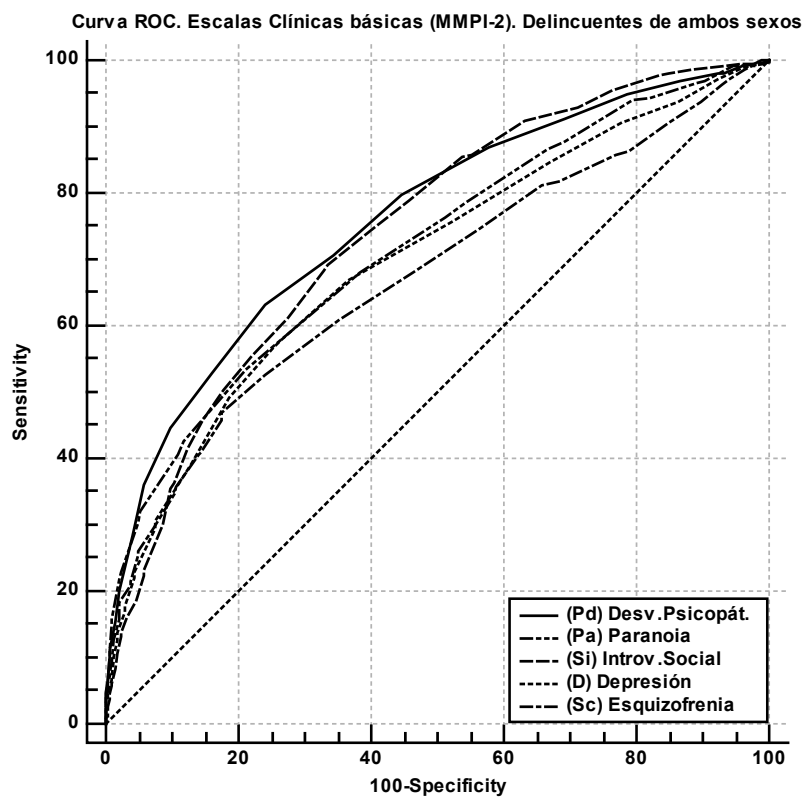

Figura 1. Precisión diagnóstica mediante la Curva ROC. Escalas Clínicas (MMPI-2). 


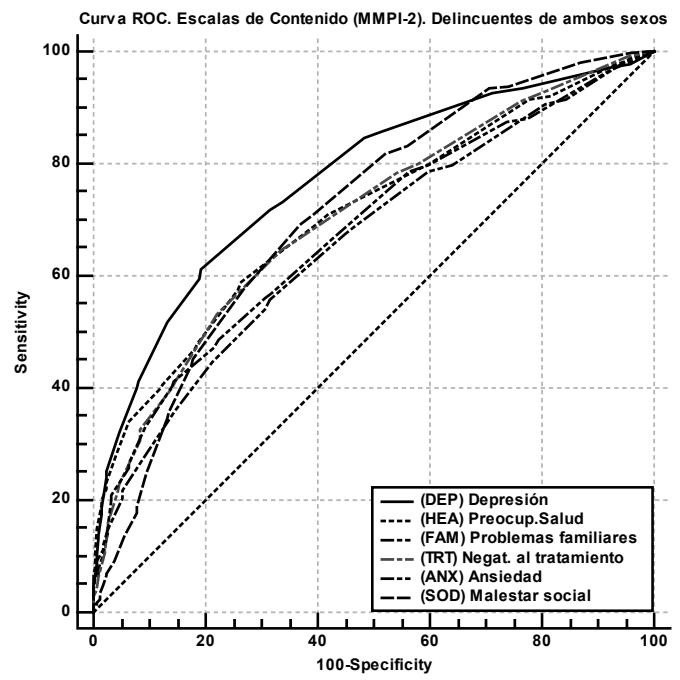

Figura 2. Precisión diagnóstica mediante la Curva ROC. Escalas de Contenido (MMPI-2).

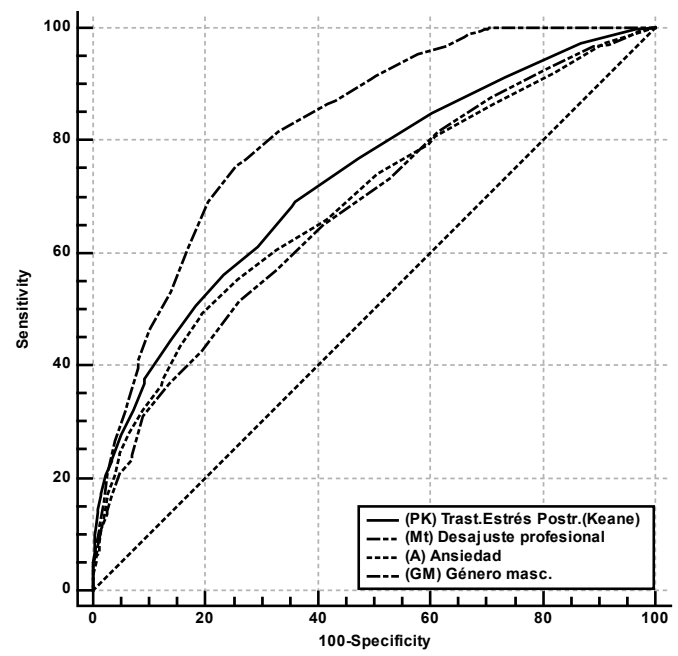

Figura 3. Precisión diagnóstica mediante la Curva ROC. Escalas Suplementarias (MMPI-2). 


\section{Análisis por género}

En la Tabla 2 se compara la precisión diagnóstica diferenciada por género. Se ha tomado como referencia que el valor de la $A B C \geq 0.70$ en las mujeres y poder comparar con el valor obtenido por los varones. Se observan dos aspectos interesantes de la precisión diagnóstica del MMPI-2: (1) ha aumentado el número de variables del MMPI-2 que han superado el límite propuesto por la $A B C(\geq 0.70)$ con respecto a las obtenidas cuando el género se evaluaba conjuntamente (Tabla 1); y (2) la existencia de una mayor precisión diagnóstica en las mujeres $(A B C$, Sensibilidad y Especificidad).

Mientras que la precisión global media, referida esencialmente por el Área Bajo la Curva $(A B C)$ de las diferentes escalas en las mujeres se aprecia en torno al 0.82 (con un rango entre 0.912 y 0.702 ), en los varones se encuentra en 0.67 (con un rango entre 0.824 y 0.521 ). Esto nos está indicando que el MMPI-2 muestra mayor precisión diagnóstica con las mujeres que con los varones.

\section{Tabla 2}

Comparación por género de la precisión diagnóstica de las variables más representativas (MMPI-2) de la personalidad delictiva mexicana

\begin{tabular}{|c|c|c|c|c|c|c|c|c|c|c|c|}
\hline \multirow{2}{*}{$\begin{array}{l}\text { Varia- } \\
\text { bles }\end{array}$} & \multicolumn{5}{|c|}{ Varones $(n=728)$} & \multicolumn{6}{|c|}{ Mujeres $(n=142)$} \\
\hline & $\mathrm{ABC} S \mathrm{SE}$ & $95 \% \mathrm{CI}$ & $\begin{array}{l}\text { P. de } \\
\text { corte }\end{array}$ & S. & E. & $\mathrm{ABC}$ & SE & $95 \%$ CI & $\begin{array}{l}\text { P. de } \\
\text { corte }\end{array}$ & S. & E. \\
\hline
\end{tabular}

Escalas Clínicas Básicas

\begin{tabular}{lllllllllllll}
$\mathrm{Pd}$ & .739 & .013 & $.715-.761$ & $>51$ & 60.9 & 74.7 & .863 & .022 & $.818-.901$ & $>51$ & 75.4 & 82.4 \\
$\mathrm{~Pa}$ & .707 & .013 & $.683-.730$ & $>53$ & 53.8 & 76.6 & .775 & .027 & $.722-.822$ & $>53$ & 51.4 & 90.1 \\
$\mathrm{Si}$ & .717 & .013 & $.694-.740$ & $>44$ & 68.1 & 64.4 & .851 & .022 & $.805-.891$ & $>41$ & 85.9 & 69.7 \\
$\mathrm{D}$ & .679 & .014 & $.654-.703$ & $>47$ & 55.9 & 71.7 & .786 & .028 & $.734-.832$ & $>49$ & 61.3 & 90.8 \\
$\mathrm{Sc}$ & .640 & .014 & $.614-.664$ & $>52$ & 44.2 & 79.7 & .824 & .025 & $.775-.867$ & $>51$ & 60.6 & 96.5 \\
$\mathrm{Pt}$ & .611 & .015 & $.585-.636$ & $>53$ & 43.5 & 74.2 & .702 & .028 & $.730-.829$ & $>54$ & 49.3 & 97.9 \\
\hline
\end{tabular}




\begin{tabular}{lllllllllllll}
\hline & \multicolumn{4}{c}{ Varones $(n=728)$} & \multicolumn{5}{c}{ Mujeres $(n=142)$} \\
$\begin{array}{l}\text { Varia- } \\
\text { bles }\end{array}$ & ABC & SE & $95 \%$ CI & $\begin{array}{l}\text { P. de } \\
\text { corte }\end{array}$ & S. & E. & ABC & SE & $95 \%$ CI & $\begin{array}{l}\text { P. de } \\
\text { corte }\end{array}$ & S. & E. \\
\hline
\end{tabular}

Escalas de Contenido

$\begin{array}{llllllllllllllll}\text { DEP } & .745 & .013 & .722-.768 & >50 & 59.9 & 77.9 & .883 & .020 & .840-.918 & >45 & 74.6 & 89.4 \\ \text { HEA } & .678 & .014 & .653-.702 & >48 & 57.0 & 70.6 & .849 & .023 & .802-.888 & >48 & 69.0 & 88.0 \\ \text { FAM } & .623 & .014 & .597-.648 & >50 & 44.0 & 75.1 & .844 & .023 & .797-.884 & >42 & 84.5 & 69.7 \\ \text { TRT } & .670 & .014 & .646-.695 & >46 & 64.4 & 61.8 & .845 & .023 & .798-.885 & >47 & 66.9 & 91.5 \\ \text { ANX } & .646 & .014 & .621-.670 & >51 & 30.74 & 83.65 & .834 & .024 & .786-.875 & >44 & 75.4 & 81.0 \\ \text { SOD } & .688 & .014 & .664-.712 & >43 & 68.0 & 60.0 & .833 & .024 & .784-.874 & >44 & 75.4 & 78.9 \\ \text { FRS } & .692 & .014 & .667-.716 & >48 & 63.0 & 66.3 & .750 & .029 & .695-.799 & >48 & 57.0 & 83.1 \\ \text { ANG } & .521 & .015 & .495-.547 & >63 & 19.0 & 88.9 & .729 & .030 & .673-.780 & >49 & 47.9 & 88.7 \\ \text { ASP } & .666 & .014 & .641-.690 & >46 & 59.3 & 64.4 & .799 & .026 & .748-.844 & >48 & 55.6 & 90.1 \\ \text { LSE } & .655 & .014 & .629-.679 & >44 & 58.1 & 66.3 & .790 & .027 & .738-.836 & >47 & 57.0 & 88.0\end{array}$

Escalas Suplementarias

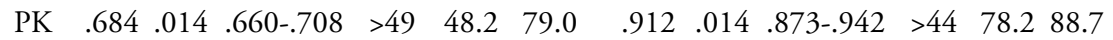

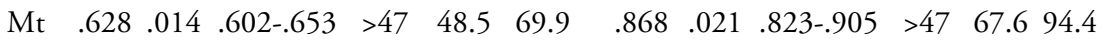

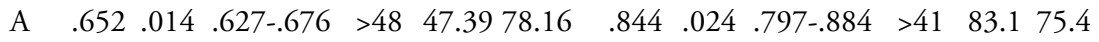

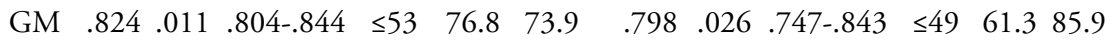

Notas: $\mathrm{ABC}=$ Área Bajo la Curva; $\mathrm{SE}=$ Error estándar; $\mathrm{CI}=$ Intervalo de confianza; P. de corte = Punto de corte; S. = Sensibilidad; E. = Especificidad. Pd = Desviación Psicopática; Pa = Paranoia; $\mathrm{Si}=$ Introversión social; D = Depresión; $\mathrm{Sc}=$ Esquizofrenia; $\mathrm{Pt}=$ Psicastenia; $\mathrm{DEP}=$ Depresión; HEA = Preocupación por la salud; FAM = Problemas familiares; TRT = Indicación negativa hacia el tratamiento; ANX = Ansiedad; SOD = Desajuste social; FRS = Miedo; ANG = Ira; ASP = Prácticas antisociales; LSE = Baja autoestima; PK = Trastorno de estrés postraumático (Keane); $\mathrm{Mt}=$ Malestar escolar; $\mathrm{A}=$ Ansiedad, GM = Rol masculino. 


\section{Discusión}

En ningún momento se ha intentado, en este estudio, describir el perfil del delincuente mexicano a través de las variables psicológicas del MMPI-2. Interesaba saber el grado de precisión diagnóstica que aporta el MMPI-2 en sus diferentes variables sobre la personalidad delictiva. Se ha optado por el análisis de la curva ROC porque dispone de los detectores fundamentales para apreciar la precisión global de una prueba diagnóstica a través de los tres elementos que la caracterizan: Área Bajo la Curva $(A B C)$, Sensibilidad $(S)$ y Especificidad $(E)$.

Es necesario dejar constancia que los resultados ofrecidos por los delincuentes de nacionalidad mexicana con las diversas escalas del MMPI-2 tienen su propia peculiaridad por dos razones fundamentalmente: a) la singularidad de la misma sociedad que la identifica y define; y b) la política penitenciaria, tomada en su más amplio sentido, (a través de los poderes Legislativo, Judicial y Ejecutivo) es peculiar y distinta a la de cualquier otro país. No es extraño, por ello, que los delincuentes mexicanos expresen también sus características psicológicas a través de la técnica del Minnesota, independiente de la coincidencia de características de la personalidad con cualquier otro país.

Generalmente, la evaluación en centros de internamiento se centra en tres cuestiones: (a) evaluación de la salud mental, (b) la evaluación de riesgos, y (c) evaluación de las necesidades (Megargee, 2006b). En este último punto de las necesidades, es importante determinar sus características y tratamiento de cada preso. El objetivo del tratamiento es maximizar la probabilidad de que los internos se inserten en su comunidad de origen. Si el sistema penitenciario tiene como función esencial la rehabilitación de los reclusos, en cada delincuente se deben valorar las necesidades educativas, profesionales, emocionales y de salud mental, formulando programas de gestión y tratamiento personificado (Megargee, 2006b).

Con los resultados obtenidos en este estudio se obtienen dos importantes conclusiones: (a) que la precisión diagnóstica aportada por el MMPI-2, se puede considerar, de forma general, como "moderada" 
(Swets, 1979), y (b) la precisión diagnóstica que aporta el MMPI-2 sobre la personalidad delictiva es distinta según sea su género.

La mayor parte de investigaciones con delincuentes empleando el MMPI-2, se han centrado en las escalas Clínicas básicas (Butcher, et al. 2015; Megargee, 2006a, 2006b). En este estudio se ha contemplado también otro conjunto de variables que evalúa el MMPI-2 (Contenido y Suplementarias) con la intención de poder aportar la precisión de otras variables importantes de la personalidad delictiva mexicana. La mayoría de los investigadores excluyen las escalas $M f$ (masculinidad-feminidad) y la $S i$ (introversión social) del grupo de escalas Clínicas básicas, sin embargo Panton (1974), que hizo gran parte de la investigación con el original MMPI en entornos correccionales de adultos, incluyó las 10 escalas "estándar". Por ejemplo, en nuestro estudio, observamos que la escala $S i$ (Introversión social), en el conjunto de delincuentes, ocupa un puesto primordial, tras la $P d$ (Desviación Psicopática) del grupo de escalas Clínicas básicas $(A B C=0.742$; Sensibilidad $=69.3$; Especificidad $=66.6$ punto de corte $=>44)$, comportándose como una de las variables que identifica la personalidad del delincuente mexicano. De manera semejante ocurre, con esta misma variable, cuando se diferencian los delincuentes por género.

La curva ROC es un gráfico en el que se observan todos los pares Sensibilidad/Especificidad resultantes de la variación continua de los puntos de corte en todo el rango de resultados observados. En el eje de ordenadas se sitúa la Sensibilidad, o fracción de Verdaderos Positivos, y en el eje X se sitúa la fracción de los Falsos Positivos (1-Especificidad) (Burgueño et al. 1995). Dependiendo de la elección del punto de corte, la Sensibilidad/Especificidad varía.

El índice obtenido por el Área Bajo la Curva $(A B C)$ nos muestra la probabilidad de clasificar correctamente un par de individuos, delincuente y no delincuente, seleccionados al azar de la población, con los resultados obtenidos al aplicarles la prueba diagnóstica. Es, en definitiva, una medida global de la exactitud de la prueba para clasificar a los individuos. Cuanto más elevada, mayor exactitud. Así, el valor de $P d=0.760$ en el conjunto de delincuentes (Tabla 1), significa que 
un individuo, seleccionado aleatoriamente del grupo de delincuentes, tendrá el $76.0 \%$ de las veces un valor mayor que un individuo elegido al azar del grupo no delincuentes. Swets (1979), interpreta la ABC: valores entre $0.5-0.7$ indican baja exactitud; entre $0.7-0.9$ pueden ser útiles para algunos propósitos; y un valor mayor de 0.9 indica exactitud alta. La capacidad de discriminación de la prueba diagnóstica puede evaluarse estimando el intervalo de confianza (CI) de la ABC: si el intervalo no incluye el valor de 0.5 , la prueba es capaz de discernir entre delincuentes y no delincuentes. Por convenio la $\mathrm{ABC}$ es siempre $\geq 0.5$ porque sus valores están comprendidos entre 0,5 (si no existen diferencias en la distribución de resultados de la prueba entre los subgrupos delincuente y no delincuentes), y 1.0 cuando existe separación perfecta entre las dos distribuciones.

Con la Sensibilidad de una prueba se obtiene la probabilidad (\%) de clasificar correctamente a un individuo cuya situación real sea definido como "positivo" (= delincuente). Detecta a los "verdaderos positivos". La Especificidad, detecta la probabilidad (\%) de clasificar correctamente a un individuo cuya situación sea definida como "negativo" (no delincuente). Detecta a los "verdaderos negativos". Es decir, siguiendo con la variable $P d$ del conjunto de internos (Tabla 1), se puede apreciar que, con el punto de corte $>51$ (en puntuaciones $\mathrm{T}$ de la adaptación mexicana del MMPI-2), nos aporta la información de que el $63.2 \%$ son verdaderos delincuentes y el resto (100-63.2 = $36.8 \%$ ) son falsos positivos, por lo cual, se puede concluir que la precisión diagnóstica de la variable Pd del MMPI-2, es considerada como "moderada" (Swets, 1979). Es de advertir que si se toma otro punto de corte diferente, el binomio sensibilidad / especificidad variará. Es decir, que el MMPI-2 con su precisión diagnóstica global 63.2 puede diagnosticar como falsos positivos en la variable $P d$ a un $36.8 \%$ de personas, supuestamente sobre una personalidad delictiva.

Como se puede apreciar por los datos aportados (Tabla 1), la precisión diagnóstica a través del Área Bajo la Curva $(A B C)$ del conjunto de los delincuentes, ofrecida por las distintas variables del MMPI-2, se encuentra de media en torno al $0.72 \%$, lo que está indicando que su 
precisión global es considerada por Swets (1979) como efectivamente moderada ya que en su intervalo de confianza no contempla el valor de 0.5 .

Aparte de las dos variables más destacadas en el perfil general de los delincuentes, el código mayormente encontrado es el 4-6 $(P d-P a)$, en este estudio se ha podido apreciar que también el MMPI-2 muestra su precisión con otras variables, como muestra la Tabla 1: la variable $G M$ (Rol de género masculino), DEP y $D$ (Depresión), Pd (Desviación Psicopática), Si (Introversión social), Pa (Paranoia), PK (Trastorno de Estrés Postraumático), SOD (Malestar social), HEA (Preocupación por la salud), TRT (Negatividad al tratamiento), que definen mejor a la personalidad delictiva mediante el MMPI-2. Sorprende en este análisis la precisión con que ha detectado el MMPI-2 el rol de género masculino $(G M)$, fundamentalmente en varones, de estas personalidades seguras de sí mismas, y que toman decisiones fácilmente, con pocos temores y experimentan poca angustia emocional, manteniendo intereses masculinos muy estereotipados y que suelen participar en actividades netamente masculinas. Igualmente la detección de las variables denotadoras de la depresión ( $D$ y $D E P$ ), la introversión social $(S i)$ de los internos y el malestar social (SOD) reflejando con ello la incomodidad ante situaciones sociales, el aislamiento, la inadaptación general, y autodesprecio.

El trastorno de estrés postraumático $(P K)$ como generador de angustia emocional, ansiedad y falta de confianza en sus propias capacidades, es otra variable del MMPI-2 que ha demostrado ofrecer una moderada precisión para diferenciar a los delincuentes de los no delincuentes. Finalmente la preocupación por la salud (HEA) es otra variable que ha detectado la técnica del test de Minnesota, posiblemente por las condiciones contextuales de las cárceles mexicanas, a veces insalubres, con deficiente atención médica o preocupación por enfermar de los mismos delincuentes, contexto que ha sido reiteradamente expuesto en las diversas encuestas del Centro de Investigación y Docencia Económicas (CIDE). 
En la Tabla 2, sobre el análisis comparativo entre varones y mujeres, se puede apreciar dos conclusiones importantes: (1) Un mayor número de variables que superan el $\mathrm{ABC}>0.70$ en las mujeres, (2) las notables diferencias entre ambos sexos, siendo, sensiblemente superiores, la precisión diagnóstica, en las mujeres. De forma resumida, el MMPI-2 presenta una mayor precisión diagnóstica global $(A B C>0.70)$ en las mujeres en las siguientes áreas y variables:

- En los síntomas de ansiedad con las variables de ansiedad (A), trastornos de estrés postraumático $(\mathrm{PK})$, obsesividad $(\mathrm{Pt})$, trastornos de ansiedad (ANX) y sentir miedo (FRS ).

- En la sintomatología psicopatológica, fundamentalmente en las variables de esquizofrenia (Sc), depresión (D) y paranoia (Pa).

- En los desajustes sociales especificados por las variables indicadoras de problemas de conducta (Pd ), problemas sociales (SOD), prácticas antisociales (ASP ), e introversión (Si ).

- Con los problemas familiares (FAM ), la baja autoestima (LSE ), y la negación a someterse a tratamiento (TRT), es superior en las mujeres.

- Sin embargo el rol de género masculino (GM ) mostró el MMPI-2 una precisión diagnóstica superior en los varones.

Este estudio presenta una limitación importante por cuanto no logra aportar datos acerca del poder preditivo positivo $(P P+)$ y del poder predictivo negativo $\left(P P_{-}\right)$de cada una de las variables más destacadas de la personalidad delictiva mexicana. El poder predictivo depende de la prevalencia de la enfermedad (de cada una de las variables del MMPI-2) no se ha encontrado estadística fiable de la población reclusa mexicana para poder ser utilizada.

La conclusión más importante de este estudio es que el MMPI-2, a través de sus distintas variables, presenta una precisión diagnóstica moderada (Swets, 1979). Es decir, el valor predictivo del MMPI-2 para 
diagnosticar la personalidad delictiva no alcanza valores óptimos, por cuanto para evaluar la personalidad delictiva se debe acompañar de información adicional aportada por diferentes instrumentos y fuentes de información, como la entrevista clínica, registros de salud física y mental e informes de casos y datos de la historia criminal, de manera integrada. Las observaciones del personal penitenciario con experiencia, deberán ocupar un lugar preferente en el conjunto de la información, aunque suele ser un recurso descuidado y minusvalorado. Tal como tiene investigado el MMPI-2 (Megargee, 2006b), las variables indicadoras de potencial violento son, precisamente, la $P d(4), P a(6), S c$ (8), $M a$ (9) y abuso de sustancias/alcohol (Butcher et al. 2015, pp. 210-211) y estas variables presentan en este estudio con el MMPI-2 una precisión considerada por Swets (1979) como moderada.

\section{Referencias}

Ampudia, R.A. (2012). Cuestionario sociodemográfico (CSD). Universidad Nacional Autónoma de México (UNAM).

Azaola, E. \& Bergman, M. (2009). Delincuencia, marginalidad y desempeño institucional. Resultados de la tercera encuesta a población en reclusión en el Distrito Federal y Estado de México. Centro de investigación y Docencia Económicas (CIDE), México

Azaola, E. \& José, C. (1996): Las mujeres olvidadas. Un estudio acerca de las cárceles para mujeres en la República Mexicana. El Colegio de México / Comisión Nacional de Derechos Humanos, México, DF.

Bergman, M., Azaola, E. \& Magaloni, A.-L. (2006). Delincuencia, marginalidad y desempeño institucional. Resultados de la segunda encuesta a población en reclusión en el Distrito Federal y el Estado de México. Centro de Investigación y Docencia Económicas (CIDE), México, DF. 
Burgueño, M.J., García-Bastos, J.L. \& González-Buitrago, J.M. (1995). La curva ROC en la evaluación de las pruebas diagnósticas. Medicina Clinica, 104, 661-670.

Butcher, J. N. (2013). 25 Highlights of Using the MMPI/MMPI-2 with Criminal Offenders. Retrieved from. Recuperado de http://www. umn.edu/mmpi

Butcher, J. N. Hess, G. A., Greene, R. L. \& Nelson, L. D. (2015). Using the MMPI-2 in Forensic Assessment. American Psychological Association (APA). https://doi.org/10.1037/14571-000

Centro de Investigación y Docencia Económicas (CIDE) (2012). Resultados de la Primera Encuesta realizada a Población Interna en Centros Federales de Readaptación Social. México.

Comisión Nacional de los Derechos Humanos (CNDH) de México (2015). Coordinación General de Comunicación y Proyectos. Recuperado de http://www.cndh.mx

Diario Excelsior (4/02/2016): "Más de 14.000 presos están por reingreso en reclusorios de la Ciudad de México. Recuperado el 29/02/2016, de http:/www.excelsior.com.mx/ comunidad/2016/02/02/1072503

Gomez-San Luis, A. \& Almanza-Avendaño, A. (2016). Impacto del narcotráfico en jóvenes de Tamaulipas, México: drogas e inseguridad. Revista de Psicología, 34(2), 445-472. https://doi. org/10.18800/psico.201602.009

Greene, R.L. (2011). The MMPI-2/MMPI-2-RF. An interpretative manual. Boston: Allyn \& Bacon.

Jiménez, F., Sánchez, G. \& Ampudia, A. (2009). La contribución de la Escala PSY-5 al MMPI-2. Revista Iberoamericana de Diagnóstico y Evaluación Psicológica, 28(2), 31-43.

Jiménez, F., Sánchez, G. \& Tobón, C. (2009). A social desirability scale for the MMPI-2. Which of the two: Wiggins (WSD-R) or Edwards (ESD)? The European Journal of Psychology Applied to Legal Context, 1(2), 147-163.

Jones, T., Beidleman, W.B. \& Fowler, R. D. (1981). Differentiating violent and nonviolent prison inmates by use of selected 
MMPI scales. Journal of Clinical Psychology, 37(3), 673-678.doi: 10.1002/1097-4679(198107)37:3\%3C673::AIDJCLP2270370340\%3E3.0.CO;2-P

Lanyon, R.I. (1993). Validity of MMPI sex offender scales with admitters and no admitters. Psychological Assessment, 5(3), 302306. doi: org/10.1037/1040-3590.5.3.302

Llamas, M. (2013). El sistema penitenciario en cifras. SinEmbargo.OPINIÓN. Fecha de acceso 25/10/2015. Recuperado de http:// www.sinembargo.mx./opinion/28-07-2013/16235

Lucio, E., Reyes-Lagunes, I. \& Scott, R. L. (1994). MMPI-2 for Mexico: Translation and adaptation. Journal of Personality Assessment, 63, 105-116. doi: 10.1207/s15327752jpa6301_9

MedCalc Statistical Software version 14.12.0 (2014). MedCalc Software bvba, Ostend, Belgium. Recuperado de http://www. medcalc.org.

Megargee, E. I. (2006a). Use of the MMPI-2 in correctional settings. En J. N. Bucther (Ed.), MMPI-2: A practitioner's guide (pp. 327360). Washington, DC: American Psychological Association (APA).

Megargee, E. I. (2006b). Using the MMPI-2 in criminal justice and correctional settings. Minneapolis: University of Minnesota Press. doi: 10.1037/0022-006X.53.6.874

Nicholson, R. A., Mouton, G. J., Bagby, M. \& Buis, T. (1997). Utility of MMPI-2 Indicators of response Distortion: Receiver Operating Characteristic Analysis. Psychological Assessment, 9(4), 471-479. doi: 10.1037/1040-3590.9.4.471

Pavelka, F. L. (1986). Psychosocial characteristics of parolees in forensic social work. Journal of Psychiatry \& Law, 14, 217-223.

Panton, J. H. (1974). Personality differences between male and female prison inmates measured by the MMPI. Criminal Justice and Behavior, 1 (4), 332-339. doi: 10.1177/009385487400100404

Pennuto, T.O. (2010). Murder and the MMPI-2: The necessity of knowledgeable legal professionals. Golden Gate University Law Review, 34(2), 340-391. 
Pérez, C. C. \& Azaola, E. (2012). Resultados de la primera encuesta realizada a población interna en Centros Federales de Readaptación Social. México: CIDE.

Sánchez, G. \& Jiménez, F. (2003). La Escala Superlativa de Butcher y Han (1995): el fingimiento en la adaptación española del MMPI-2. Revista de Psicología, XXI(1), 5-39.

Sánchez, G., Jiménez, F. \& Ampudia, A. (2008). Detectando el perfil simulador en el MMPI-2: una propuesta basada en la investigación. Revista de Psicología, XXVI(2), 277-298.

Secretaría de Gobernación (Enero, 2013). Estadisticas del Sistema Penitenciario Nacional. Recuperado el 2 de julio de 2013, de http://www.ssp.gob.mx/portalWebApp/ShowBinary? nodeId=/ BEA\%20 Repository/365162//archivo

Secretaría de Gobernación (Julio, 2015). Incidencia delictiva del Fuero Común 2015. Secretariado Ejecutivo del Sistema Nacional de Seguridad Pública (SESNSP). Centro Nacional de Información. México.

Secretaría de Seguridad Pública del Gobierno Federal de los Estados Unidos Mexicanos (Octubre de 2011). Cuaderno de Estadisticas del Sistema Penitenciario Federal del Órgano Administrativo Desconcentrado Prevención y Readaptación Social.

Solís, L. Buen, N. \& Ley, S. (2013). La cárcel en México: ¿para qué? México evalúa, Centro de Análisis de Políticas Públicas.

Spaans, M., Barendregt, M., Muller, E., de Beurs, E., Nijman, H. \& Rinne, T. (2009). MMPI profiles of males accused of severe crimes: A cluster analysis. Psychology, Crime \& Law, 15(5), 441450. doi: 10.1080/10683160802356704

Steffan, J., Morgan, R.D., Lee, J. \& Sellbom, M. (2010). A comparative analysis of MMPI-2 malingering detection models among inmates. Assessment, 17(2), 185-196. doi: $10.1177 / 1073191109359382$

Swets, J.A. (1979). ROC analysis applied to the evaluation of medical imaging techniques. Investigative Radiology, 14(2), 109-121. doi: 10.1097/00004424-197903000-00002 
Swets, J.A. \& Pickett, R. M. (1982). Evaluation of diagnostic systems: methods from signal detection theory. New York: Academic Press.

Villanueva, C. R. (2015). La sobrepoblación en los centros penitenciarios de la República Mexicana. Análisis y pronunciamiento. Comisión Nacional de los Derechos Humanos. Comunicado de prensa CGCP/310/15. Recuperado de http://www.cndh.mx.

Zweig, M. H. \& Campbell, G. (1993). Receiver-operating characteristic (ROC) plots: A fundamental evaluation tool in clinical medicine. Clinical Chemistry, 39(4), 561-577.

Recibido: 02 de abril, 2016 Revisado: 24 de mayo, 2016 Aceptado: 21 de junio, 2016 